\title{
Deep Rule-Based Approach for the Classification of Wagon Bogie Springs Condition
}

\author{
Carlos M. Viriato Neto \\ Graduate Program in Electrical Engineering \\ Federal University of Juiz de Fora \\ Juiz de Fora, Brazil \\ viriato_ee@hotmail.com
}

\author{
Luca G. Honorio \\ Undergraduate Program in Mechanical Engineering \\ Federal University of Juiz de Fora \\ Juiz de Fora, Brazil \\ luca.garcia@engenharia.ufjf.br \\ Eduardo P. de Aguiar \\ Department of Industrial and Mechanical Engineering \\ Federal University of Juiz de Fora \\ Juiz de Fora, Brazil \\ eduardo.aguiar@engenharia.ufjf.br
}

\begin{abstract}
This paper focuses on the new model of classification of wagon bogie springs condition through images acquired by a wayside equipment. As such, we are discussing the application of a deep rule-based (DRB) classifier learning approach to achieve a high classification of a bogie, and check if they either have spring problems or not. We use a pre-trained VGG19 deep convolutional neural network to extract the attributes from images to be used as input to the classifiers. The performance is calculated based on the data set composed of images provided by a Brazilian railway company. The presented results of the report demonstrate the relative performance of applying the DRB classifier to the questions raised.

Index Terms-DRB, defects, pre-trained VGG19, wagon bogie springs, image processing.
\end{abstract}

\section{INTRODUCTION}

Since the emergence of steam-powered machines, rail transportation has become an effective solution for connecting urban centers, as well as a low-cost alternative to the industries' transactions. Due to these circumstances, the wagons are submitted to stressing cycles with heavy loads, increasing their bogie defects through springs fatigue.

In this context, image processing and computational intelligence techniques are increasingly participating in the solution for this scenario. Since their capacity to detect critical wagon conditions enables to guarantee the safety production and high productivity of this system of these transportation systems.

$\mathrm{Gu}$ and Angelov, in [1] introduced deep rule-based (DRB) classifiers [2]-[4]. The DRB classifier is a general approach that serves as a strong alternative to current deep neural network (DNN) [2]-[4]. It is non-parametric, non-iterative, highly parallelizable and computationally efficient; it achieves very high classification rates, surpassing other methods [4].
Moreover, it further extends the DRB classifier [1] with a self-organising, self-evolving semi-supervised learning strategy by exploiting the idea of "pseudo label" naturally with its prototype-based nature.

To deal with the classification of wagon bogie springs conditions, this paper discusses the DRB algorithm during the classification process to classify if they are or are not in bad condition.

Due to the transparency of the DRB classifier, we emphasize that the process is easy to interpret by human specialists, which is very important to present technologies for the railway sector.

The main contributions of this work are summarized below:

- The study of the wagon bogie springs conditions through DRB has never been addressed before.

- We use a pre-trained vgg-verydeep-19 deep convolutional neural network (VGG19) [5] to extract the attributes of the images. By this, the proposed model can learn abstract resources and obtain higher precision.

- We present the performance analysis in terms of the classification accuracy using a data set with images acquired from railway wayside equipment.

And our major conclusions are:

- The use of VGG19 as a feature extractor is effective for this application.

- The DRB classifier achieved mean accuracy greater than $96 \%$.

- Through the obtained results, the DRB classifier proved to be an excellent alternative for the classification of wagon bogie springs conditions. It can assist in the inspection process of the road by reducing inspection times, ensuring greater reliability and availability of the road. 
The rest of the paper is organized as follows: Section 2 deals with the formulation of the problem. Section 3 aims to discuss the concept of DRB. Section 4 discusses the results of computer simulations. Section 5 presents the main conclusions.

\section{Problem Formulation}

The bogie suspension springs are a fundamental part of the wagon's damping set which has the function of dissipating the energy caused by some unwanted vertical movements that occur in railway dynamics.

As wagon bogies springs are the main part of suspension and damping, the classification of its condition is a critical development to assist the railway companies in verifying its railway conditions and safety along with granting higher services reliability.

In 2017, 11 wagons of a 33 wagon freight train traveling north of Ely have derailed as presented in the Figure 1 . The derailment accident occurred by ineffective damping on the wagon bogies [6]. The line was blocked, affecting passenger services from Peterborough and Cambridge to Stansted Airport and London for 7 days. Rail experts say the cost of the derailment could top $£ 1.0$ million [7].

As shown in the Figure 2, a similar accident already occurred 10 years before when a line was closed for six months as result of derailment caused by bogie's suspension problems [6], [8]. The river Ouse had been shut to traffic and Network Rail had to create a $1.3 \mathrm{~km}$ access route to the site to recover the stricken wagons that were left teetering over the river Ouse [9], [10]. In addition, Network Rail had to rebuild the rail bridge across the River Ouse which cost around $£ 9.0$ million [11].

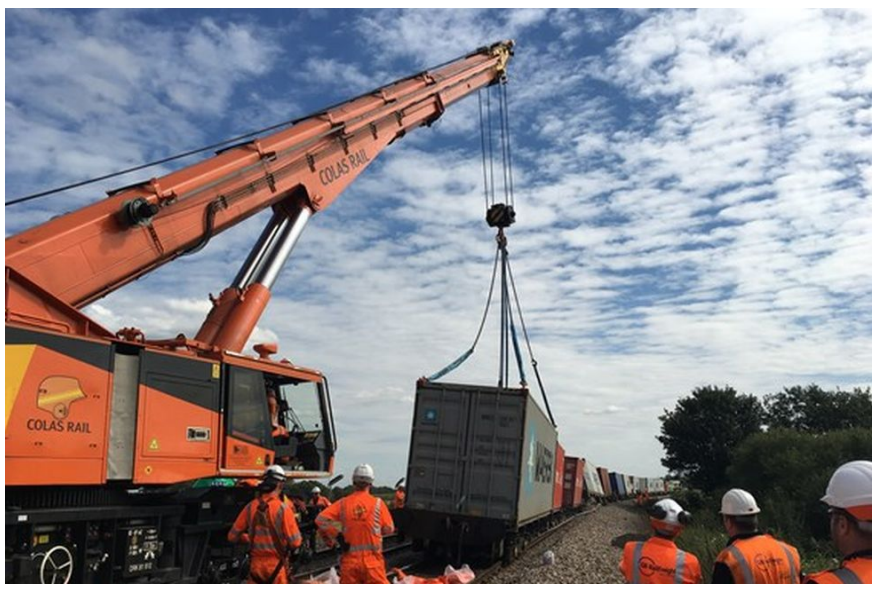

Fig. 1. A crane's lifting the derailed wagons in Cambridgeshire [12]

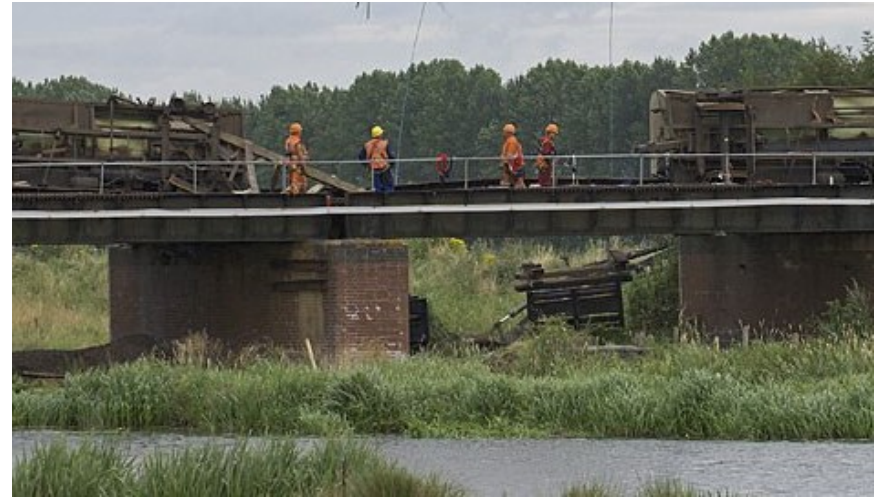

Fig. 2. The derailed wagons of freight train on Ely rail bridge [13]

According to ANTF (Brazilian National Association of Railway Transporters) [14], the volume of goods transported by the railroads increased by $95 \%$ in the period from 1997 to 2019.

In 2019, approximately US $\$ 600$ million was invested, allowing for a significant growth in the rolling stock fleet. In 1997, the railroads had 1,154 locomotives and in 2019, they already totaled 3,405 units, representing an increase of $195 \%$. In the same period, the number of wagons went from 43,816 to 115,434 , representing an increase of $163 \%$.

This trend causes an increase in speed and loads transported, changing the dynamic wheel-rail contact, thus increasing the probability of bogie spring defected. Defects in the springs occur due to different reasons, for example, as a result of fatigue, due to repetitive passages over the rail components, such as welds, joints, and switches, or due to the impacts of defect rail surface. If bogie spring defected grow and are delayed, they can lead to high maintenance costs. Therefore, rapid and automatic defect detection is essential.

Accordingly, the proposed model can reduce the impact of overhauls on trains operation, since it enables a preventive maintenance routine, which makes it possible for interventions to be carried out only when anomalies are observed.

Based on perspective, it is extremely necessary, especially because it results in order to:

- Prevent accidents caused by unexpected failures;

- Reduce the number of unproductive hours in maintenance;

- Eliminate the manual process of visual inspection;

- Reduce the number of recurrent preventive interventions;

- Reduce the probability of brinelling on a bearing;

- Increase the productivity of rail operations, given the reduction in frequency and time of operational maintenance;

- Reduce the TST (Time Stopped Train) index through the increase in the meantime between failures (MTBF). 


\section{The PROPOSAL: DRB}

DRB architecture is shown in Figure 3 . The input $\mathbf{I}$ consists of a railhead image and is subject to a normalization of pixel values up to a range of $[0,255][15]$. Subsequently, the image is scaled to $227 \times 227$ pixels to increase generalization and reduce computational complexity [15].

The VGG19 is used for resource extraction due to its simpler structure and better performance. The extracted data are processed by the fuzzy rule-based (FRB) layer, which constitutes a massively parallel set of nebulous irrigations of the AnYa type 0-order fuzzy [16], which is the basis of the DRB classifier. Finally, the decision-making classifies the images based on the degree of similarity with the prototypes generated in the training stage.

The use of a pre-trained deep convolutional neural networks to extract global vectors of image features to train generic classifiers is an alternative widely used since it allows the classifier to learn more abstract and discriminative attributes of high level obtaining greater precision [15], [17].

In this work, the VGG19 is used to vectors extracted from the wagon bogie spring images that have a dimension of $[1,4096][17]$.

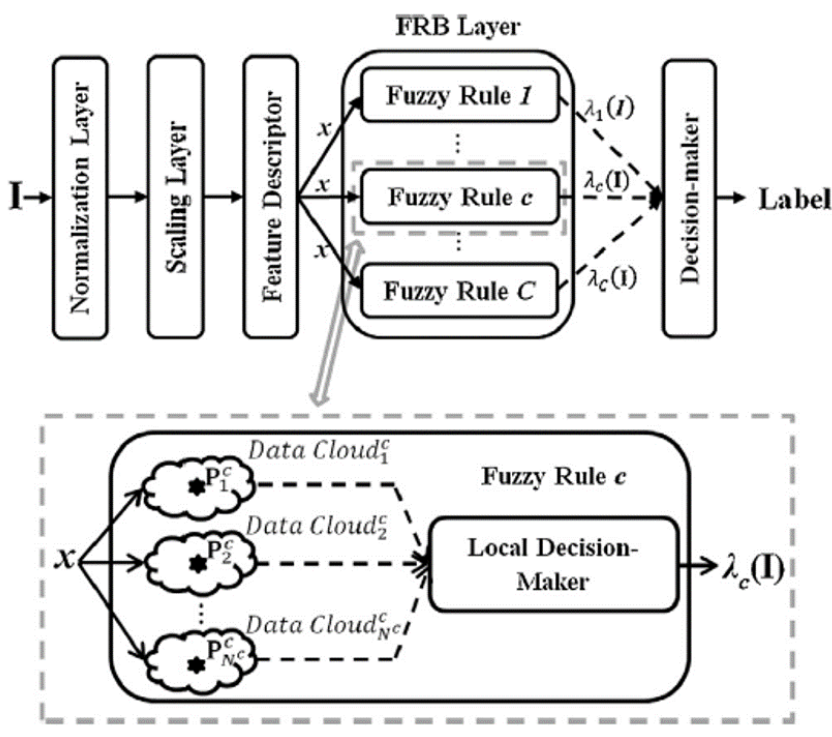

Fig. 3. Architecture of the DRB classifier [1]

A previously mentioned FRB layer is the learning mechanism of the DRB classifier. The FRB subsystems are independent of each other and can be changed without influencing the others.

Furthermore, each FRB subsystem contains a set of massively parallel fuzzy rules, formulated around generalized prototypes $P$ or learned from the corresponding class segments.
As they all have the same consequence can be combined through the logical connector " $O R$ ", as follows:

$$
\begin{array}{r}
\text { IF }\left(I \sim P_{1}^{c}\right) O R\left(I \sim P_{2}^{c}\right) O R \ldots O R\left(I \sim P_{N^{c}}^{c}\right) \\
\text { THEN }(C L A S S C)
\end{array}
$$

In Eq. 1] " " denotes similarity; $c=1,2, \ldots, C ; N^{c}$ is the number of prototypes of the $c^{\text {th }}$ class.

The prototypes, generated from data density data, based on prototypes such as fuzzy rules are generated, but due to the large dimensions of vectors $X_{1}^{c}\left(X_{1}^{c}=X_{1,1}, X_{1,2}, \ldots, X_{1, M}\right)$ it is necessary to use a cosine dissimilarity, as described below [18]:

$$
\begin{array}{r}
d(x, y)=\sqrt{2-2 \cos \theta_{x, y}} \\
=\sqrt{2-2 \frac{\sum_{i=1}^{M} x_{i} y_{i}}{\|x\|\|y\|}} \\
=\left\|\frac{x}{\|x\|}-\frac{y}{\|y\|}\right\| \\
\cos \theta_{x, y}=\frac{(x, y)}{\|x\|\|y\|}=\frac{\sum_{i=1}^{M} x_{i} y_{i}}{\|x\|\|y\|}
\end{array}
$$

$$
\|x\|=\sqrt{(x, y)}=\sqrt{\sum_{i=1}^{M} x_{i}^{2}}
$$

For this paper it was adopted $M=4096$. The Eq. 2 is important because it facilitates the computational efficiency by allowing recursive calculation.

The DRB training process is mentioned in [4] in which, the DRB classifier identifies prototypes of the segments of the observed images of each class in an autonomous and nonparametric way and forms clouds of data around the prototypes of similar segments of the same class.

In this way, the $C$ rules of massively parallel diffuse parallel $C$ order of the type AnYa in total are formed (learned) through the training processes independently, based on the identified prototypes.

The detailed training process for the FRB subsystems is described in [2] and [19], and the main procedure of the training process is summarized in pseudocode form, as shown in the Algorithm 1.

Once the training process is completed, the classification of new images can be performed using the identified FRB. 
Algorithm: Training process of the FRB subsystem

While new feature vector $x_{k}$ of the $c^{t h}$ class is available i. $x_{k} \longleftarrow \frac{x_{k}}{\left\|x_{k}\right\|}\left(\left\|x_{k}\right\|\right.$ is the norm of $\left.x_{k}\right)$;

ii. If $(k=1)$ Then

1. $\mu_{c} \longleftarrow x_{k}$ ( $\mu_{c}$ is the global mean)

2. $N_{c} \longleftarrow 1 ; p_{c, 1} \longleftarrow x_{k} ; S_{c, 1} \longleftarrow 1 ; r_{c, 1} \longleftarrow r_{0}$;

( $N_{c}$ is the number of prototypes; $p_{c, 1}$ is the first prototype; $S_{c, 1}$ is the corresponding support; $r_{c, 1}$ is the radius, $r_{0}=\sqrt{\left.2-2 \cos \left(\frac{\pi}{6}\right)\right)}$

iii. Else

1.Update global mean; $\mu_{c} \longleftarrow(k-1) \frac{\mu_{c}}{k}+\frac{x_{k}}{k}$;

2.Calculate the density of $x_{k}$ :

$$
D\left(x_{k}\right)=\frac{1}{1+\frac{\left\|x_{k}-\mu_{c}\right\|^{2}}{1-\left\|\mu_{c}\right\|^{2}}}
$$

3. Update the densities of prototypes $\left(j=1,2, \ldots, N_{c}\right)$;

$$
D\left(x_{k}\right)=\frac{1}{1+\frac{\left\|p_{c, j}-\mu_{c}\right\|^{2}}{1-\left\|\mu_{c}\right\|^{2}}}
$$

4. If $\left(\min \left(D\left(p_{c, j}\right)\right) \leq D\left(x_{k}\right) \leq \max \left(D\left(p_{c, j}\right)\right)\right)$ Then; -Find nearest prototype: $p_{n, c}=\operatorname{argmin}\left(\left\|x_{k}-p_{c, j}\right\|\right)$; -If $\left(\left\|x_{k}-p_{c, n}\right\|>r_{c, n}\right)$ Then;

$1 ; r_{c, N_{c}} \longleftarrow r_{0}$

* $N_{c} \longleftarrow N_{c}+1 ; p_{c, N_{c}} \longleftarrow x_{k} ; S_{c, N_{c}} \longleftarrow$

\section{Else}

$* p_{c, n} \longleftarrow S_{c, n} \frac{p_{c, n}}{\left(S_{c, n}+1\right)+\frac{x_{k}}{S_{c, n}+1}}$

$* S_{c, n} \longleftarrow S_{c, n}+1 ; r_{c, n}^{2} \longleftarrow \frac{1}{2}\left(r_{c, n}^{2}+\left(1-\left\|p_{c, n}\right\|^{2}\right)\right)$;

\section{End If}

5.Else

$N_{c} \longleftarrow N_{c}+1 ; p_{c, N_{c}} \longleftarrow x_{k} ; S_{c, N_{c}} \longleftarrow 1 ; r_{c, N_{c}} \longleftarrow$ $r_{0}$

\section{End If}

\section{iv. End If}

v. Generate/update the AnYa type fuzzy rule;

\section{End While}

As shown in [1], during the validation process, each test image receives a confidence score from the fuzzy rules identified in the training stage [4]:

$$
\gamma^{c}(I)=\max \left(\exp \left(-d^{2}\left(x, p_{i}^{c}\right)\right)\right)
$$

As a result, for each testing image, a vector of $1 \times C$ dimensional scores of confidence/degrees of closeness to the nearest prototypes (one per class) is generated:

$$
\gamma(I)=\left[\gamma^{1}(I), \ldots, \gamma^{C}(I)\right]
$$

The label of this testing image is decided by using the "winner-takes-all" principle [4]:

$$
\text { Label }=\operatorname{argmax}\left(\gamma^{c}(I)\right)
$$

\section{EXPERIMENTAL RESULTS}

Based on the theory addressed in the previous topic, tests were carried out to evaluate the classification model proposed in this work.

The database used in this application is made from wagons bogies of Brazilian railway company MRS Logística S.A. [20]. Such database is composed of images that are captured by a railway wayside equipment fixed to the MRS railroad in order to capture wagons bogies images each time the train passes through the site.

The images are taken from both wagon sides when it is crossing through the equipment and the examples of the images contained in the database are shown in the Figures 4 and 5. In addition, it is important to mention that the wagons are empty when they pass through the site, i.e., it means the springs expected standard condition is that they are not compressed.

Therefore, in case of Figure 4 the springs have no elastic reserve problems and It is noted because the springs have space between the turns. However, in Figure 5 is shown no space between the turn what is a critical defect because they do not have damping capacity anymore.

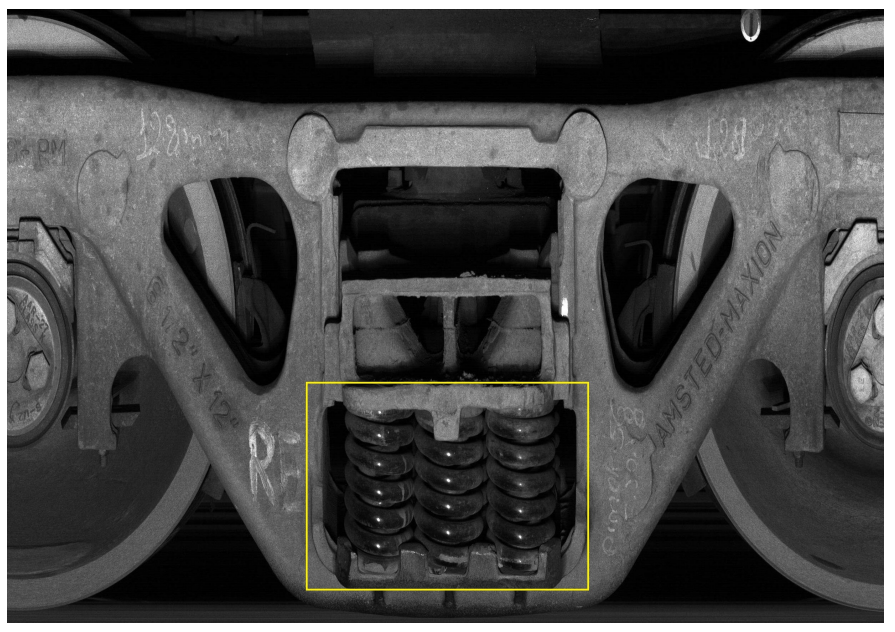

Fig. 4. Bogie springs without defect [21] 


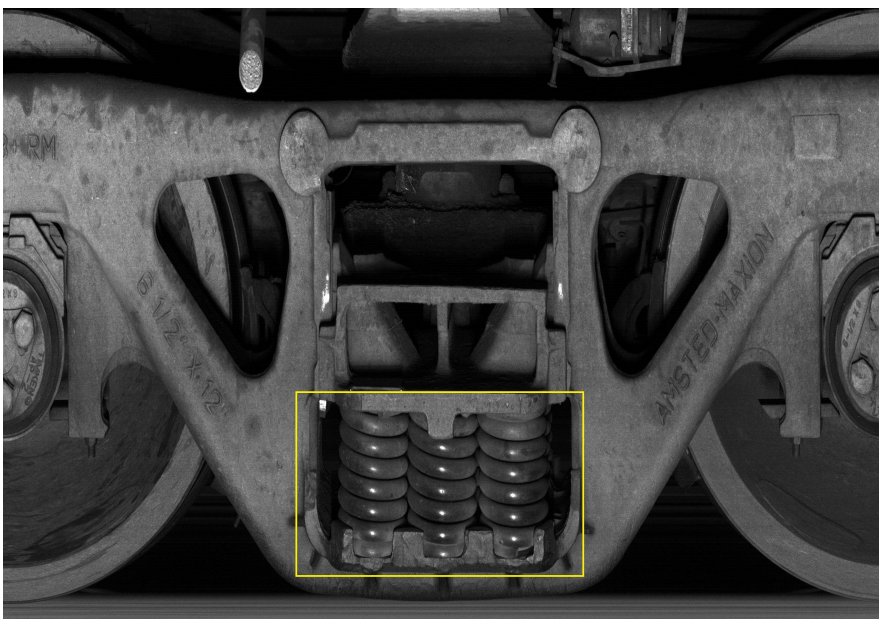

Fig. 5. Bogie springs with bad condition 21

The types of bogie that can be used on freight wagons may vary according to the type of wagon. Nonetheless, for each type of bogie the springs type and its geometric distribution are particular to each bogie type. Therefore, for the present work we used bogie images that correspond to the most representative portion of the main transport flow of MRS Logística S.A.

Taking into account the scenario previously exposed in Section III], this work aims to classify the two main conditions that can occur with the wagon bogie springs: springs with elastic reserve (without defect) and springs without elastic reserve (with bad condition), as presented in Figure 4 and Figure 5 respectively.

The experiment was conducted by collecting 250 images, of which 125 presented wagon bogie with springs without elastic reserve problems and 125 images in which correspond to bogie with elastic reserve problems.

The images used were collected evenly distributed over different periods of the day for 4 months. In addition, we distributed the data set for the test phase in an equal and random way, with the premise of $70 \%$ of the database for training and $30 \%$ for testing.

All simulations were performed in the MATLAB 2018 environment running with an Intel Core i5-7200U CPU at $2.50 \mathrm{GHz}$ with 8000MB DDR4 2700MHZ. The operating system was Windows 10 64-bit. All data were presented for the classifier 200 times.

The results presented in Figure 6 and the Table 1 show the performance of the DRB. In addition, to reducing computational complexity with the use of the VGG19, four metrics for extracting image attributes presented in Table $\Pi$ were used to measure performance during the training and test phases, respectively: Accuracy, Mean Square Error (MSE), Cohen's kappa coefficient [22] and F-score [23].
TABLE I

CONFUSION MATRIX FOR OVERALL RESULTS

\begin{tabular}{|c|c|c|}
\hline Predicted $\backslash$ Actual & $\begin{array}{c}\text { \% of Bogie springs } \\
\text { without defect }\end{array}$ & $\begin{array}{c}\text { \% of Bogie springs } \\
\text { with bad condition }\end{array}$ \\
\hline $\begin{array}{c}\text { \% of Bogie springs } \\
\text { without defect }\end{array}$ & $\mathbf{4 7 . 6 7 1} \pm \mathbf{( 1 . 3 2 4 )}$ & $2.329 \pm(1.324)$ \\
\hline $\begin{array}{c}\text { \% of Bogie springs } \\
\text { with bad condition }\end{array}$ & $1.066 \pm(1.341)$ & $\mathbf{4 8 . 9 3 4} \pm(\mathbf{1 . 3 4 1})$ \\
\hline
\end{tabular}

TABLE II

PERFORMANCE METRICS FOR OVERALL RESUlTS

\begin{tabular}{|c|c|}
\hline Test Accuracy & Test MSE \\
\hline $0.9661 \pm(0.0152)$ & $0.0339 \pm(0.0152)$ \\
\hline Test Kap & Test F-Score \\
\hline $0.9656 \pm(0.0154)$ & $0.9321 \pm(0.0305)$ \\
\hline
\end{tabular}

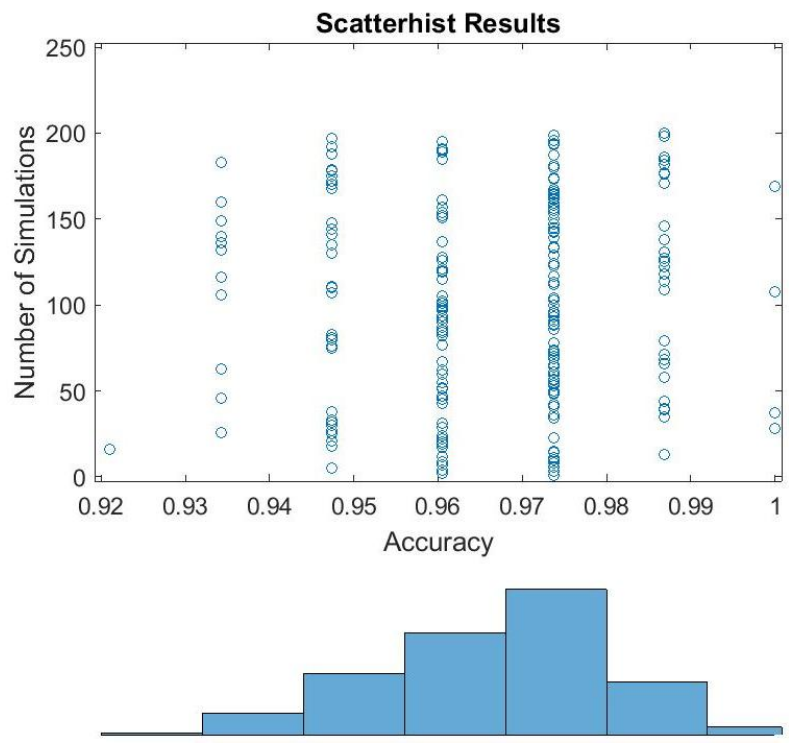

Fig. 6. Results

\section{CONClusion}

The work discusses the application of image processing techniques and computational intelligence by introducing the DRB classifier to the analysis of its results in the classification of wagon bogie springs. VGG19 was used for preprocessing the images, which proved to be very effective in extracting attributes from images due to its simple structure and better performance.

In addition, the model presented in this paper has other advantages that are not covered by classical methods, such as a learning process that is easy to interpret by a specialist; online or offline training; the ability to classify images outside the sample. 
Detecting and classifying the condition of bogie springs can be done in the present context, generating a significant reduction in cost and time. It is therefore worth mentioning that the use of intelligent systems can support decisionmaking processes, increasing the flexibility and efficiency of the process.

In this respect, the DRB classifier is an attractive alternative to quickly and efficiently diagnosing and classifying the condition of the wagon bogie springs, reducing costs and time spent on inspection.

As future work, we intend to implement the ability to learn new classes and becoming a self-evolving process by semisupervisor DRB. Furthermore, to confront the model we intend to simulate the noise conditions by simulating errors in images add noises on acquired image conditions, thereby making the DRB challenged. Also, we intend to improve the process by researching more efficient techniques for pre-processing and image classification.

\section{ACKNOWLEDGMENT}

The authors acknowledge the MRS Logística S.A. for the essential support during this work. The authors also thank the financial support of CNPq (grant 433389/2018-4), FAPEMIG (APQ-02922-18).

\section{REFERENCES}

[1] X. Gu, P. P. Angelov, Semi-supervised deep rule-based approach for image classification, Applied Soft Computing 68 (2018) 53-68.

[2] P. Angelov, X. Gu, Mice: Multi-layer multi-model images classifier ensemble, in: 2017 3rd IEEE International Conference on Cybernetics (CYBCONF), IEEE, 2017, pp. 1-8.

[3] P. Angelov, X. Gu, A cascade of deep learning fuzzy rule-based image classifier and svm, in: 2017 IEEE International Conference on Systems, Man, and Cybernetics, 2017, pp. 746-751.

[4] X. Gu, P. P. Angelov, C. Zhang, P. M. Atkinson, A massively parallel deep rule-based ensemble classifier for remote sensing scenes, IEEE Geoscience and Remote Sensing Letters 15 (3) (2018) 345-349.

[5] M. Mateen, J. Wen, S. Song, Z. Huang, et al., Fundus image classification using vgg-19 architecture with pca and svd, Symmetry 11 (1) (2019) 1.

[6] Commuters face train delays for days ITV News Anglia (August 2017).

URL https://www.itv.com/news/anglia/2017-08-15/ commuters-face-train-delays-for-days

[7] Cost of freight train derailment could top $£ 1$ million ITV News Anglia (August 2017).

URL https://www.itv.com/news/anglia/update/2017-08-18/ cost-of-freight-train-derailment-could-top-1-million/

[8] Derailed freight train near ely causes chaos in the east BBC News (August 2017).

URL https://www.bbc.com/news/uk-england-cambridgeshire-40935930

[9] Removal of derailed train resumes BBC News (July 2007). URL http://news.bbc.co.uk/2/hi/uk_news/england/cambridgeshire/ 6283186.stm

[10] Freight wagons lifted from ely rail bridge NetworkRail Media Centre (July 2007)

URI

https://www.networkrailmediacentre.co.uk/news/ freight-wagons-lifted-from-ely-rail-bridge

[11] Construction of new ely rail bridge begins NetworkRail Media Centre (October 2007).

URL

https://www.networkrailmediacentre.co.uk/news/ construction-of-new-ely-rail-bridge-begins

112] Crane moves in to remove derailed ely freight train BBC News (August 2017).

URL https://www.bbc.com/news/uk-england-cambridgeshire-40950072
[13] Wagon lift on ely rail bridge begins (July 2007). URL https://www.networkrailmediacentre.co.uk/resources/wagon-lift1

[14] O setor ferroviário de carga brasileiro ANTF - Informações Gerais (2019).

URL https://www.antf.org.br/informacoes-gerais/

[15] K. Simonyan, A. Zisserman, Very deep convolutional networks for largescale image recognition, arXiv preprint arXiv:1409.1556 (2014).

[16] P. Angelov, R. Yager, A new type of simplified fuzzy rule-based system, International Journal of General Systems 41 (2) (2012) 163-185.

[17] G. Xia, J. Hu, F. Hu, B. Shi, X. Bai, Y. Zhong, L. Zhang, Aid: A benchmark dataset for performance evaluation of aerial scene classification. arxiv 2016, arXiv preprint arXiv:1608.05167.

[18] X. Gu, P. P. Angelov, D. Kangin, J. C. Principe, A new type of distance metric and its use for clustering, Evolving Systems 8 (3) (2017) 167177.

[19] P. P. Angelov, X. Gu, Deep rule-based classifier with human-level performance and characteristics, Information Sciences 463 (2018) 196213.

[20] MRS LogÍstiCA S.A., Available in: https://www.mrs.com.br

[21] MRS LogísticA S.A., https://www.mrs.com.br MRS logística S.A. wagon bogies images database, DATABASE is not public available (2020).

[22] S. Stehman, Estimating the kappa coefficient and its variance under stratified random sampling, Photogrammetric Engineering and Remote Sensing 62 (4) (1996) 401-407.

[23] M. Sokolova, N. Japkowicz, S. Szpakowicz, Beyond accuracy, f-score and roc: a family of discriminant measures for performance evaluation, in: Australasian joint conference on artificial intelligence, Springer, 2006, pp. 1015-1021. 